This item was submitted to Loughborough's Research Repository by the author.

Items in Figshare are protected by copyright, with all rights reserved, unless otherwise indicated.

\title{
A composite material with Poisson's ratio tunable from positive to negative values: an experimental and numerical study
}

PLEASE CITE THE PUBLISHED VERSION

http://dx.doi.org/10.1007/s10853-013-7666-1

\section{PUBLISHER}

(C) Springer

VERSION

NA (Not Applicable or Unknown)

\section{PUBLISHER STATEMENT}

This work is made available according to the conditions of the Creative Commons Attribution-NonCommercialNoDerivatives 4.0 International (CC BY-NC-ND 4.0) licence. Full details of this licence are available at: https://creativecommons.org/licenses/by-nc-nd/4.0/

\section{LICENCE}

CC BY-NC-ND 4.0

\section{REPOSITORY RECORD}

Hu, Hong, Vadim V. Silberschmidt, and Xiaonan Hou. 2019. "A Composite Material with Poisson's Ratio Tunable from Positive to Negative Values: An Experimental and Numerical Study”. figshare. https://hdl.handle.net/2134/25647. 


\title{
A composite material with Poisson's ratio tunable from positive to negative values: an experimental and numerical study
}

\author{
Hong Hu • Vadim Silberschmidt
}

\begin{abstract}
The Poisson's ratio describes an extent of transverse deformation of a material when an axial strain is applied. A change of the Poisson's ratio from positive to negative can equip a material with a set of specific properties. In this paper, a study of a composite material with a tunable Poisson's ratio is presented. Samples of such a composite were first fabricated with two different polymers based on a composite structure proposed in our previous work using a multi-material additive-manufacturing system. Using both experimental and numerical methods, deformation mechanisms and mechanical properties of this composite material were analyzed. The obtained results demonstrate that its Poisson's ratio can be reduced by increasing the difference in stiffness of constituent materials and turned from positive to negative when this difference is sufficiently high. Additionally, the study also validates a possible method to fabricate composites with designed structures and multi-constituent materials using additive-manufacturing techniques.
\end{abstract}

\section{Introduction}

The Poisson's ratio describes an extent of transverse behavior of a material when it is loaded axially. Most traditional materials have a positive Poisson's ratio. However, there is a strong research interest in developing

\section{H. Hu $(\bowtie)$}

Institute of Textiles and Clothing, The Hong Kong Polytechnic University, Hung Hom, Hong Kong

e-mail: tchuhong@polyu.edu.hk

V. Silberschmidt

Wolfson School of Mechanical and Manufacturing Engineering, Loughborough University, Loughborough, UK and studying engineering materials with negative Poisson's ratios (NPR) [1-6] and zero Poisson's ratios (ZPR) [7-9].

Especially, NPR materials drew recently significant attention, because of their unusual properties and multiple potential applications in different areas, such as textile [1013], automotive [14], military [15], biomedical [9], aerospace $[16,17]$ industries, etc. At present, the NPR effect is generally achieved in cases of specific microstructures of materials. The deformation mechanisms of these structures cause transversal shrinking (under axial compression) or expansion (under tension) of the overall material, resulting in the overall NPR [5]. Although some progress has been made, there are still two significant challenges in the development of NPR and ZPR materials. The first is a fundamental design of NPR materials. To date, most of the NPR materials are studied based on certain re-entrant, chiral structures, and rotating units $[5,18-20]$. These basic units are usually periodically and symmetrically assembled [17, 21-24], according to their deformation mechanism and orientations to provide the NPR effects. Although these materials could extend the NPR behavior of the basic units to the overall materials, the overall NPR effects are limited to certain directions. Such an orientation dependence is the reason that most of the current studies are still performed in a two-dimensional space. So far, there are only a few studies focusing on the NPR effects in 3D. Lakes [4, 25] first reported a 3D foam structure with the NPR effect. He then published a well-known idealized 3D re-entrant structure with a complicated tetrakaidecahedron structure [6]. Based on a 3D rotational structure, Alderson and Evans [26] developed analytical models for tetrahedral framework silicates. However, these studies focused only on certain cellular materials with highly heterogeneous porous microstructures. As the developed theories are based on complex porous microstructures, they are hardly used for 
other engineering materials and do not achieve good mechanical properties.

The second challenge is the fabrication of NPR or ZPR materials. The current state of research for most NPR materials is still limited to a theoretical stage. Besides cellular solids and some fiber-reinforced composites, there are a few successful NPR materials that can be easily manufactured and employed in a real engineering environment [14, 27-29]. It is because most NPR materials usually have some embedded structures with intricate geometries. As a result, it is very hard to use traditional manufacturing techniques to fabricate NPR materials, especially in a 3D space. With the fast development of additive-manufacturing methods, some studies were performed to fabricate NPR materials using these techniques $[23,24]$. Although only a mono-material has been used to fabricate NPR materials based on the well-known NPR structures, the efforts have proven the potential of these techniques to fabricate 2D and 3D NPR materials.

In our previous study [30], a novel composite material was introduced theoretically, based on a structure with inclusions randomly distributed in a matrix. Due to the randomness of distribution, the composite structure could exhibit an in-plane isotropic NPR effect. For that structure, the Poisson's ratio of the composite could be changed from a positive value to a near-zero or even a negative one if suitable geometry and material properties of constituents are used. Since the effect of randomness is not orientationdependent, the fundamental concept could potentially be extended to a 3D space. In order to validate this concept in $2 \mathrm{D}$, composite samples were first fabricated using two polymers with different stiffness. Rubber material with higher stiffness was used to form inclusions while the other with lower stiffness was used as matrix. Both experimental tests and numerical calculations were then conducted to analyze a compression behavior of this composite material. The effect of mechanical properties of constituent materials on the Poisson's ratio and effective (global) properties of the composite material are discussed on the basis of the obtained results.

\section{Fabrication of composite samples}

To fabricate the samples, Object Connex ${ }^{\mathrm{TM}} 3 \mathrm{D}$ Printer was used in this study. Using a PolyJet ${ }^{\mathrm{TM}}$ technology, the system could combine different materials in a 3D printed model during the same print job. The geometry model is designed using CAD software and exported as a STL file, which approximates the design using triangular facets. Then the 3D printer reads the STL file and fabricates successive layers of the specimen by depositing certain materials at defined areas.
The obtained composite sample is shown in Fig. 1a. It contains 80 re-entrant triangle inclusions embedded in a matrix. The overall dimensions of the sample are $100 \mathrm{~mm}$ (along $X$ axis, see Fig. $1 \mathrm{a}) \times 120 \mathrm{~mm}(Y) \times 20 \mathrm{~mm}(Z)$, and the respective dimensions of the testing area are $100 \mathrm{~mm} \times 100 \mathrm{~mm} \times 20 \mathrm{~mm}$ (Fig. 1b). Locations of inclusions were randomly generated and their orientation distribution was uniform. Due to the triangle shape, the rotation range of inclusions is $120^{\circ}$, and it was divided into 8 intervals. Within each interval, ten random values of rotation angle were selected. The geometrical features of a re-entrant inclusion are shown in Fig. 1c. The gray area is the struts of the inclusion, and the area inside the struts is a void space. The distance $(L)$ between two vertices and strut thickness $(T)$ of the inclusion were $10 \mathrm{~mm}$ and $0.2 \mathrm{~mm}$, respectively. The degree of re-entrant inclusion is controlled by parameter $\mathrm{H}$, which was selected as $0.5 \mathrm{~mm}$ in this study. The constituent materials used are two different kinds of polymer rubber-like materials-TangoBlackPlus $^{\text {TM }}$ FLX9850 and FLX9895-indicated as material A and material B. Their strain-stress curves measured in uniaxial compression tests are shown in Fig. 2. Since material A has lower stiffness, it was used to fabricate matrix; and material B was used for inclusions. Both kinds of materials are incompressible, with a hyperelastic behavior; their Poisson's ratios are 0.5. Using the multimaterial additive-manufacturing system, material $\mathrm{A}$ and material B were combined during the manufacturing process, with the thickness of each horizontal layer of the composite being about $16 \mu \mathrm{m}$.

\section{Uniaxial compression tests}

To analyze deformation mechanisms of the composite material, uniaxial compression tests were carried out coupled with image capture. The INSTRON universal tester 5566 was used to obtain the strain-stress relationship of the composite material. In each test, the specimen was fixed with a pair of specially manufactured clippers (Fig. 1b). The testing speed was set at $25 \mathrm{~mm} / \mathrm{min}$. Snapshots were taken using a digital camera at $2 \%$ strain increments of compression strain that was changed from 0 to $20 \%$. The obtained images would form a basis for analysis of the deformation mechanisms of the overall composite as well as its internal structure, such as the deformation of single inclusions. To eliminate the effects of heterogeneity of the specimen and of the boundary constraints due to the clippers, only displacements at the middle part of the specimen were used to calculate the overall Poisson's ratio. The dimensions of these parts were chosen as $100 \mathrm{~mm}$ (along $X) \times 12 \mathrm{~mm}(Y) \times 20 \mathrm{~mm}(Z)$. So, two horizontal parallel lines with an interval of $12 \mathrm{~mm}$ in the middle part of the 


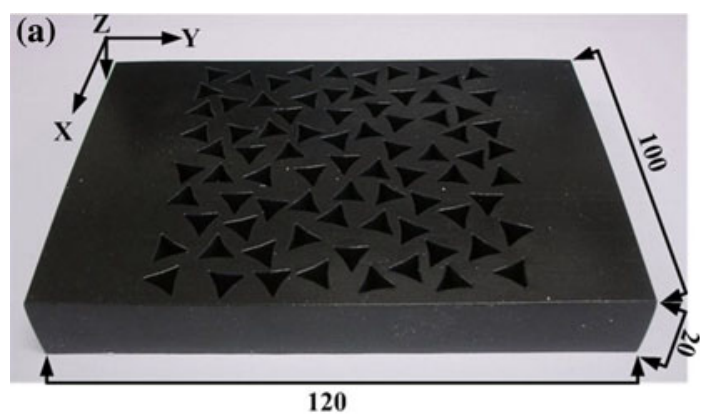

(b)

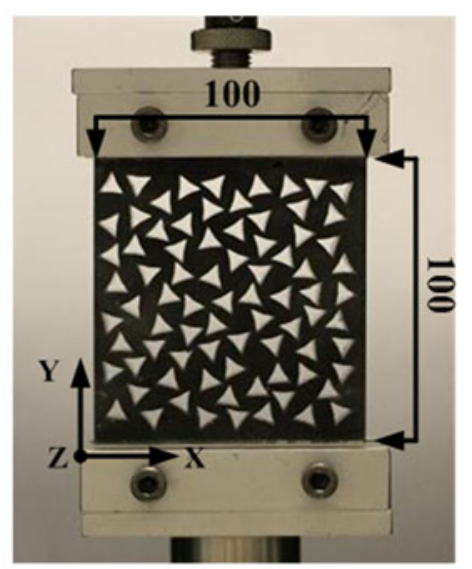

(c)

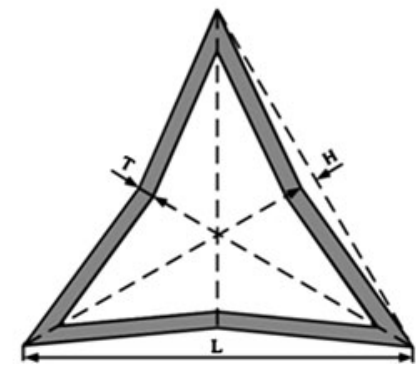

Fig. 1 Composite sample (a), testing setting (b), and geometrical features of inclusion (c)

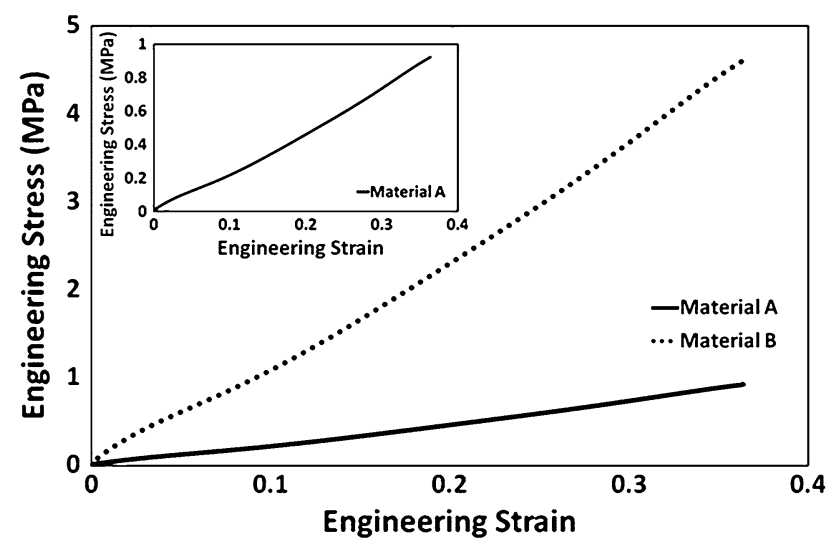

Fig. 2 Stress-strain curves of constituent materials

two lateral surfaces $(y-z)$ were drawn as marks to measure transverse displacements of the specimen. Each transverse displacement was measured with $2 \%$ strain increments of the global compression strain. For each increment, the average values of seven measurements were used to calculate the overall transverse strain of the material. Finally, the overall Poisson's ratio $v$ of the composite structure was calculated with the following equation.

$v=-\varepsilon_{\mathrm{t}} / \varepsilon_{\mathrm{v}}$

where $\varepsilon_{\mathrm{v}}$ and $\varepsilon_{\mathrm{t}}$ are the averaged transverse strain and axial compression strain, respectively.

\section{Finite element analysis}

The main approach to develop finite element (FE) models of NPR materials was discussed in the previous paper [30]. In this study, the same approach is used. However, the FE models developed in this paper are to simulate a real experiment, with a relatively large in-plane compression deformation (engineering strain of $20 \%$ ) of real composite samples with considerations of both geometry nonlinearity of the overall composite structure and material nonlinearity of the constituent materials. The geometry model was developed using a programming language Python, and the $\mathrm{FE}$ analysis was performed with a commercial FE software ABAQUS. The constituent materials were treated as hyperelastic isotropic materials according to the experiment results (Fig. 2), and their material models were defined using the Ogden material model. Their Poisson's ratios were 0.5. Elements CPS4R were used to mesh the matrix and Elements CPS3 were used for struts of inclusions. The boundary conditions of the model were set up to simulate the compression test of the composite material. To describe constraints applied by the designed clippers, the bottom of the composite sample was fixed with all the degrees of freedom constrained. A uniform displacement in the negative $y$-direction $(y=-20 \mathrm{~mm})$ was applied to the top of the modeled units to simulate static compression deformation. Meanwhile, the contacts between internal surfaces of the deformed inclusions were also taken into account in the simulation.

In this study, three different models were developed with the same geometric structure but with different material definitions. As shown in Table 1, model A-B was developed to simulate the compression behavior of the fabricated composite specimen. Real material properties were used to define the constituents of the composite. As mentioned above, the matrix area was of material $\mathrm{A}$ and the struts of inclusions were of material $\mathrm{B}$, in which stiffness is about five times higher than that of material A. This model was used for comparison with the experimental results to validate the basic concept. To analyze the effects of the difference in stiffness levels of the constituent materials on the deformation behavior and mechanical properties of the 
Table 1 Constituent materials in FE models

\begin{tabular}{llll}
\hline Model & A-B & A-A & A-100A \\
\hline Material for matrix & A & A & A \\
Material for struts of inclusions & B & A & $100 A$ \\
\hline
\end{tabular}

composite structure, models A-A and A-100A were also developed. In model A-A, the same material (material A) was used for both the matrix and struts of inclusions, simulating a composite fabricated using only one type of material. In model A-100A, a much stiffer hyperelastic material, which was assumed to be 100 times stiffer than material A (material 100A), was used for the struts of inclusions. Its Poisson's ratio was also 0.5.

The stress-strain relationship for each model was obtained by calculating the reaction forces and displacements at the upper surface of the structure. The averaged transverse strain, which was obtained by averaging the levels of transverse strains calculated from the transversal displacements of nodes located at the left- and right-hand sides of the middle part of the structure, was used to calculate the overall Poisson's ratio of the composite material. The number of nodes selected at each side was seven, and the distance between two adjacent nodes was $2 \mathrm{~mm}$, reproducing the measurement method used in compression experiment.

\section{Results and discussion}

Figure 3 shows the Poisson's ratio as a function of compression strain obtained from the experiments and in simulation $\mathrm{s}$ with FE model A-B. For the reference, the Poisson's ratios of the constituent materials, which are 0.5, are also plotted. It is apparent that both the composite specimen and FE model exhibit a significant reduction in their overall Poisson's ratio, compared with that of their constituent materials, although the calculated results are somewhat higher than those in experiments. Especially, a near-ZPR (about 0.007) was obtained for the composite specimen in the tests. Analyzing internal deformations of both the FE model and the composite sample, it can be found that the reduction of the Poisson's ratio of the latter comes from the closure of the re-entrant inclusions (Fig. 4). When an external compression loading was applied to it, the internal re-entrant inclusions started to close dragging the surrounding matrix with them. As a result, the overall composite material tended to contract laterally instead of expanding.

From the results of model A-B, it can be seen that the Poisson's ratio slowly decreases with the increment of compressive strain. The Poisson's ratio decreases by

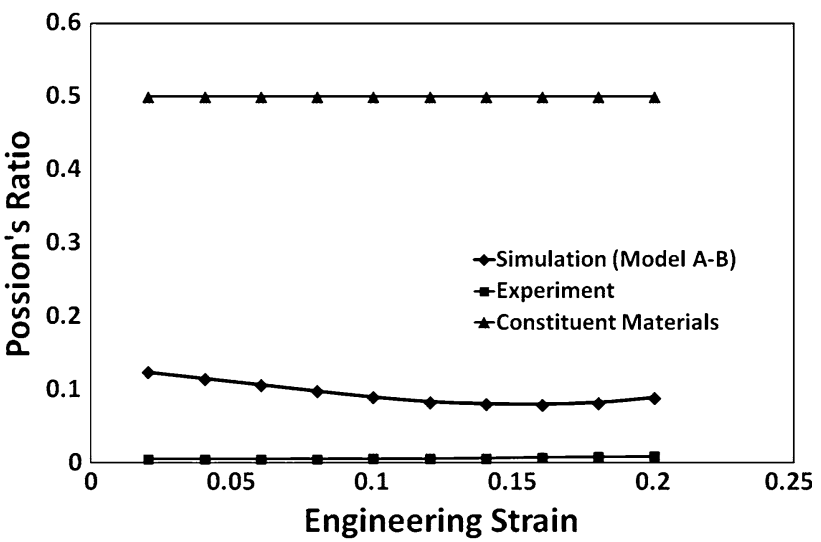

Fig. 3 Poisson's ratio as a function of compression strain for constituent materials, FE results and experiments

$27.4 \%$ when the overall compression strain increases from 0.020 to 0.107 . After that, the Poisson's ratio keeps almost constant until the compression strain reaches 0.2. Considering the average Poisson's ratio calculated for the whole range of compression strains with the FE model, which is 0.095 , it is about $81 \%$ lower than that of its constituent materials (i.e., 0.5). This result means that the proposed composite structure can effectively reduce the overall Poisson's ratio of the composite material even when the constituents are incompressible materials with a high Poisson's ratio. The results from the experiment show a much lower overall Poisson's ratio than that calculated with the FE model. In the experimental results, the Poisson's ratio is almost constant in the entire range of strains. Its magnitude is about 0.007 , which is $98.6 \%$ lower than that of the constituent materials. The difference between the experimental data and simulations results may be caused by the material formulation in the FE analysis and measurement precision of experiments.

The stress-strain curves obtained with the FE model A-B and in compression tests are presented in Fig. 5. A nonlinear behavior can be observed at the initial stage of the deformation (strain $\leq 0.015$ ) in the experimental results; this differs from a linear performance predicted with the FE model. This difference may arise from adjustment of the specimen in the fixture of the experimental set up. Due to very low indentation hardness of the rubber-like materials used in this study, the interaction between the clippers and specimen could provide additional constraints at the initial stage. Shear stresses can also occur in the clipping area of the specimen. Still, after a further strain increase to 0.02 , both curves collapse, with a good agreement between the experimental and simulation results up to strain of 0.1 . Beyond this point, an obvious difference is observed between the experiment and calculations, growing with the increase in compressive strain. When the overall strain reaches 0.2 , the calculated stress is $19.5 \%$ higher than the 
Fig. 4 Deformation of composite structure under compression
FE Model

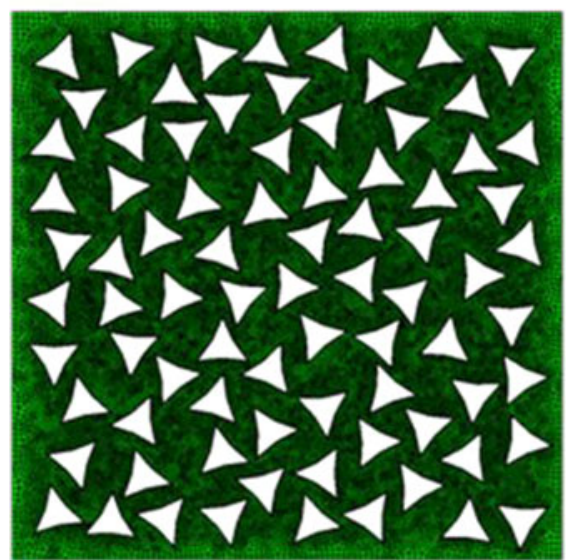

Experiment

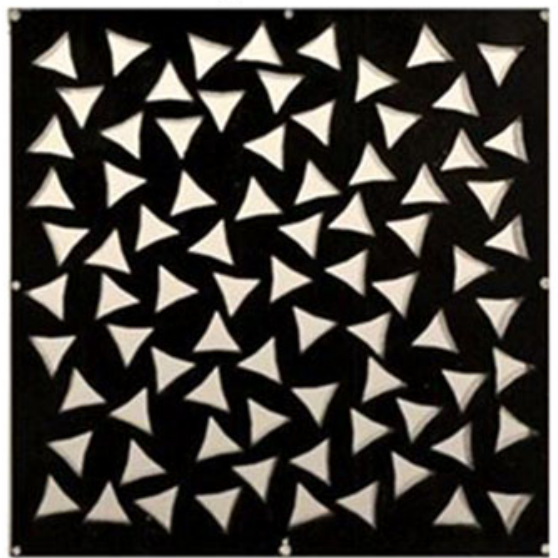

Engineering Strain 0\%
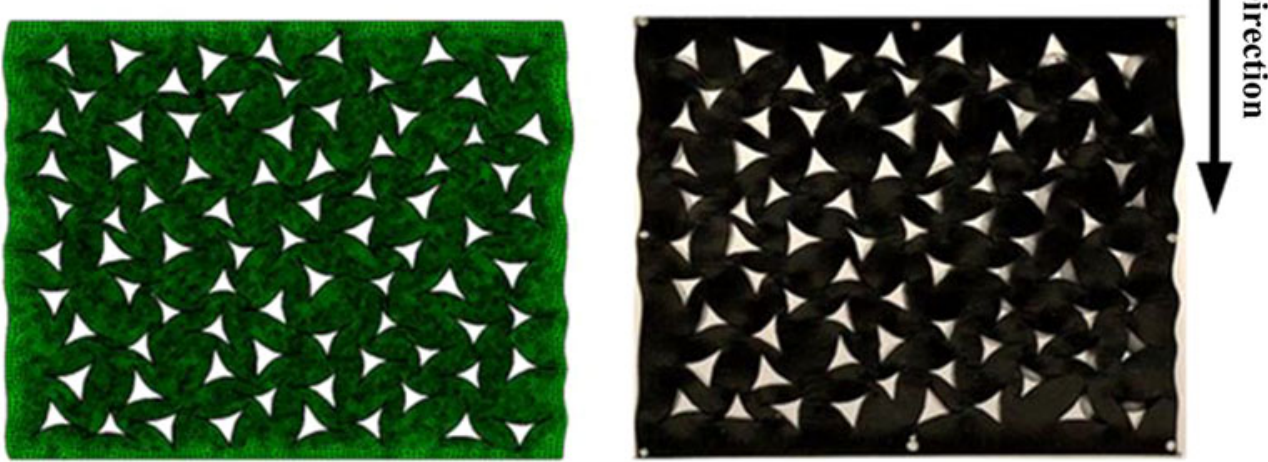

Engineering Strain 20\%

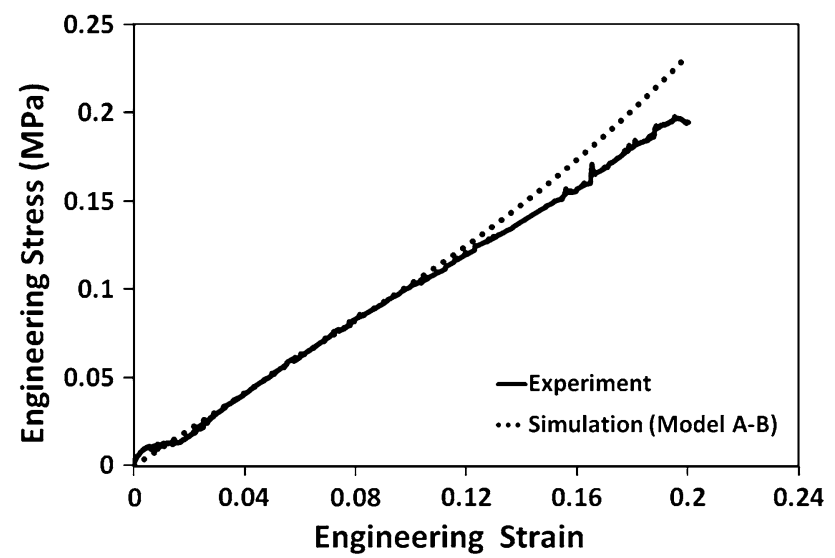

Fig. 5 Comparison of experiment and calculated stress-strain curves

measured level. This difference may originate from local buckling in some parts of the specimen, reducing its total load-bearing capacity.

Based on the above comparison between the FE model A-B and test results for composite specimens, it could be concluded that the developed FE method is generally capable to describe the deformation behavior of this kind of composite structures. Therefore, a further comparison was

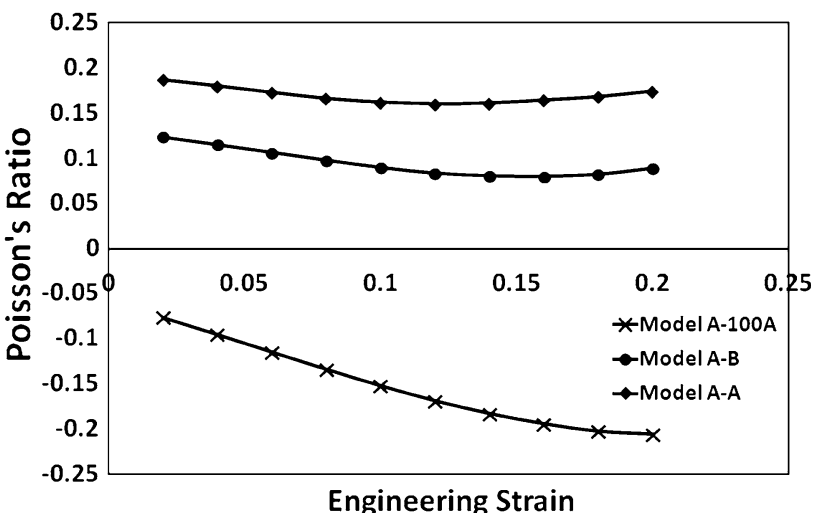

Fig. 6 Poisson's ratio as a function of compression strain calculated with different FE models

undertaken for various FE models with the same geometry of inclusions but various mechanical characteristics of constituents. The results of such comparison are presented in Fig. 6. Obviously, the Poisson's ratio of the composite structure is reduced with increasing stiffness of inclusions' struts. When the struts of inclusions have the properties of material A, which is the material of the matrix, the average Poisson's ratio of the overall composite structure is 0.17 . 
This value is much lower than the Poisson's ratio of the constituent materials (0.5). When the struts of the inclusion are defined by material $\mathrm{B}$, which is about 5 times stiffer than material A, the average Poisson's ratio is further reduced to 0.095 , which is $44.1 \%$ lower than that of model A-A. An increase in stiffness of struts to the 100-fold of material A causes a transition of the overall Poisson's ratio to a negative value. These results indicate that the difference in stiffness of constituents can significantly affect the deformation behavior of the composite structure and the increase of this difference can cause a change in the sign of its Poisson's ratio. It should be noted that the reduction of the Poisson's ratio is caused by the closure of voids of reentrant inclusions in the composite structure. As the stiffer
Fig. 7 Closure of inclusions in test specimen and different FF models under compression

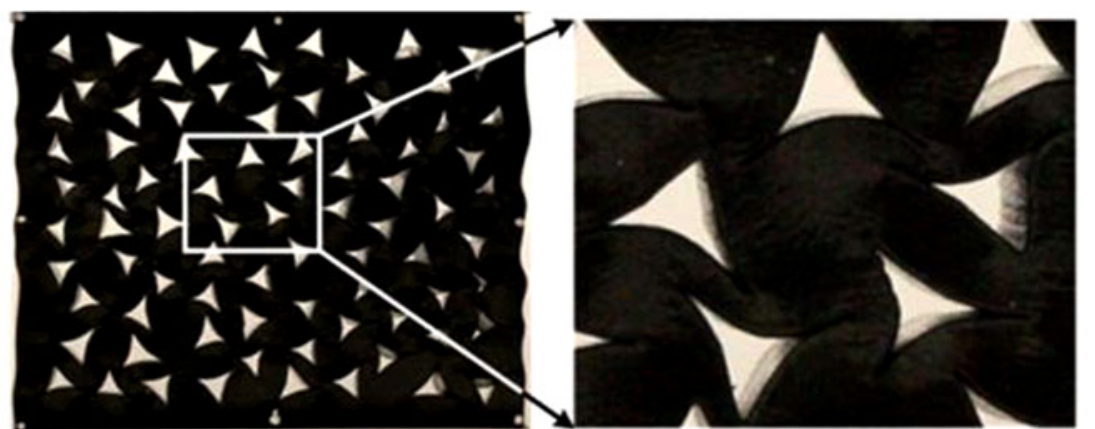

Deformed Specimen and local deformation of inclusions

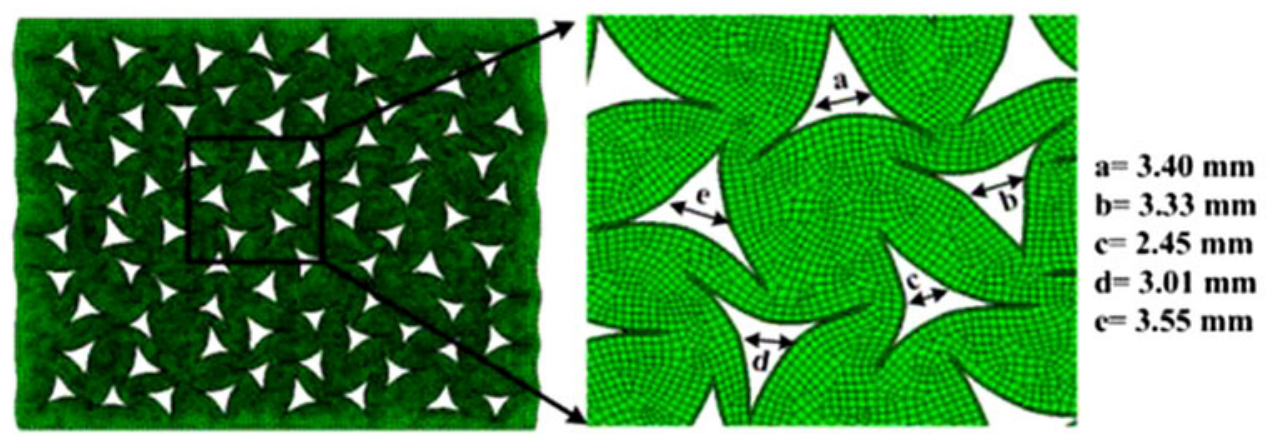

Deformed Model A-B and local deformation of inclusions

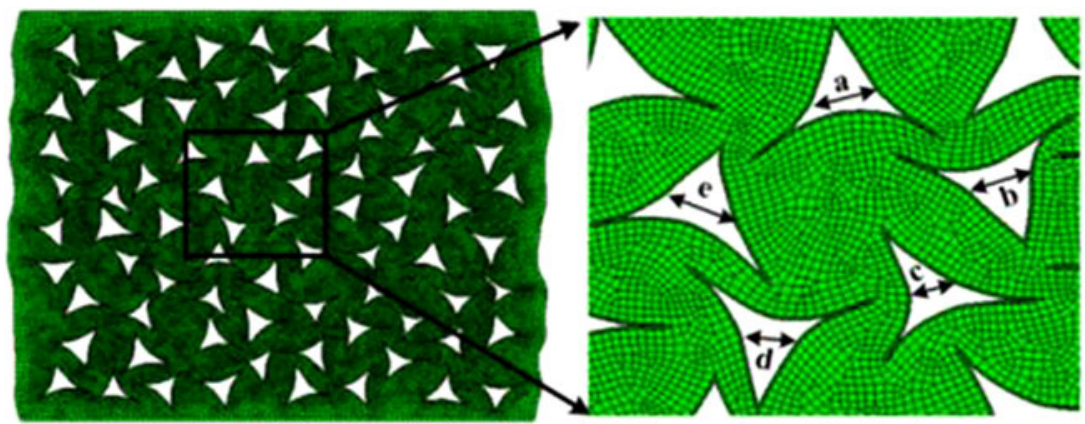

$\mathrm{a}=3.85 \mathrm{~mm}$ $b=3.63 \mathrm{~mm}$ $c=2.45 \mathrm{~mm}$ d $=3.52 \mathrm{~mm}$ $\mathrm{e}=4.11 \mathrm{~mm}$

\section{Deformed Model A-A and local deformation of inclusions}

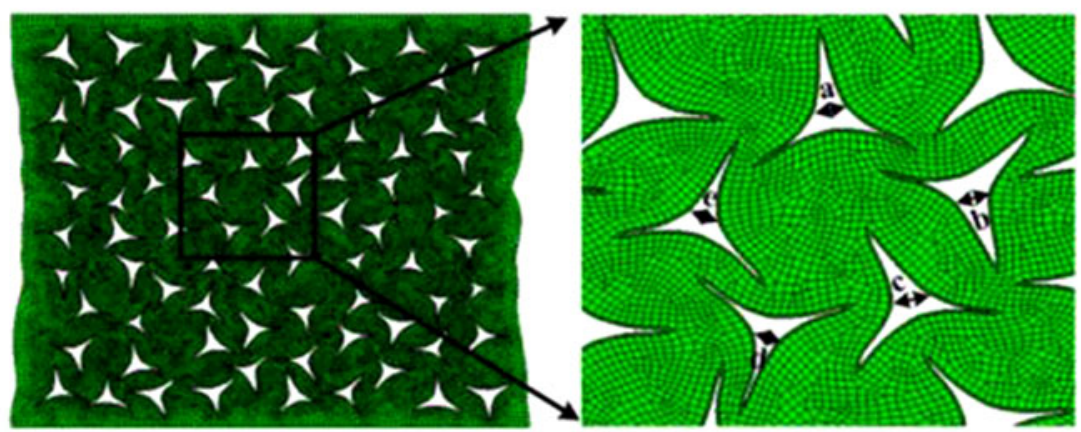

$\mathrm{a}=1.87 \mathrm{~mm}$ $\mathrm{b}=2.10 \mathrm{~mm}$ c $=1.90 \mathrm{~mm}$ d= $1.59 \mathrm{~mm}$ $\mathrm{c}=1.73 \mathrm{~mm}$

Deformed Model A-100A and local deformation of inclusions 


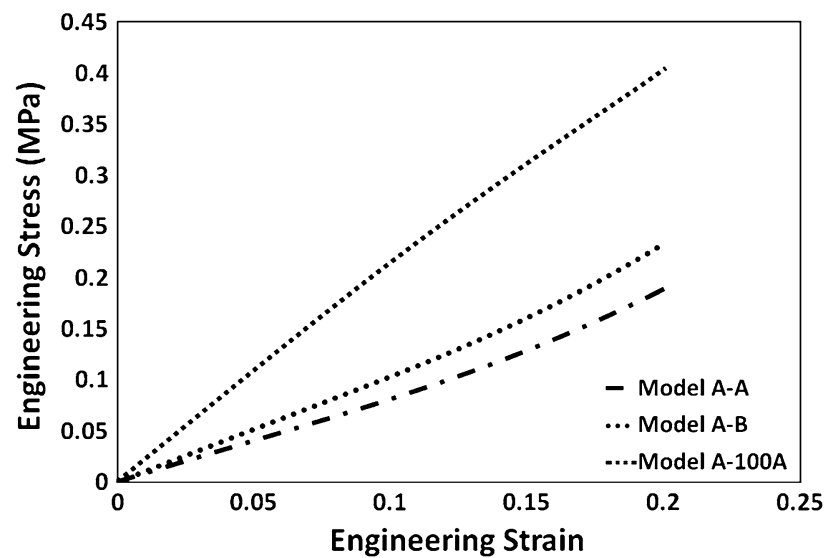

Fig. 8 Effective stress-strain curves of different FE models

struts cause a more pronounced closing effect, a lower and even NPR can be obtained. In Fig. 7, five inclusions chosen for each FE model demonstrate the effect of different stiffnesses of constituent materials on the closure behaviors of inclusions together with experimental observations. For a better comparison, the distances measured between two internal vertexes of each inclusion shown in the pictures are given near each image; a smaller value means a better closure. Apparently, stiffer inclusions have a stronger closure effect than softer ones as more matrix material is dragged by the struts of inclusions to the voids, leading to a higher reduction in the overall Poisson's ratio of the composite materials. These results indicate that the Poisson's ratio of the composite structure can be tuned by changing the stiffness of constituent materials.

The stress-strain curves of the composite structures calculated with different FE models are shown in Fig. 8. It is noticeable that the composite material with stiffer inclusions exhibits a significantly stiffer overall mechanical response, even though the volume fraction of inclusions' material was only $4.6 \%$ of the overall composite structure. The reinforcing effect caused by stiffer inclusions agrees with the results for the composite structures with linearelastic constituent materials in our previous study [30].

\section{Conclusions and remarks}

The concept of the composite structure with NPR was introduced in our previous study. However, only theoretical analysis was performed there, limited to the case of perfect linear-elastic material formulations for constituents. In this paper, real-life composite samples with randomly embedded re-entrant inclusions were fabricated employing a multi-material additive-manufacturing system and analyzed using experimental methods. The obtained results were used to validate the developed FE models. Additionally, a contrast in mechanical properties of constituent materials was studied as a very important and effective factor controlling the overall mechanical properties of the developed composite. The results demonstrate that the Poisson's ratio of the composite structure could be changed from a positive value to a negative one by increasing the stiffness of material used for inclusions (for the same stiffness of the matrix), simultaneously increasing the overall stiffness of the composite.

The following conclusions can be drawn from this study:

(1) The concept of developed composite structure was validated by experiment results for constituents with nonlinear material properties. The tests demonstrated that the composite material tended to shrink laterally under quasi-static compressive loading; even if its constituents are highly nonlinear incompressible materials (i.e., with high Poisson's ratios). The observed effect was caused by the closure of inclusions, which were fabricated from relatively stiffer materials. Moreover, the randomness of the inclusions' assembly determined in-plane mechanical isotropy, which is important for further studies of 3D reinforcement.

(2) The multi-material additive-manufacturing system can be used to fabricate the developed composite material, consisting of two kinds of constituents with different materials: matrix and randomly arranged inclusions. In the case of 2D composite structure, the inclusions are re-entrant polygons with internal voids, made of a material stiffer than matrix. As the struts of the inclusions are formed with very thin walls and the inclusions should be embedded in the matrix with strong bonding with it, it is very difficult to use traditional manufacturing technologies to fabricate this kind of composite structures. The successful manufacturing of the composite samples with the tunable Poisson' ratio in this study confirms the possibility of fabricating NPR or ZPR composite materials with multi-material constituents and complicated internal 2D and 3D structures.

(3) The FE analysis provides a useful tool to analyze the deformation mechanisms and mechanical properties of the suggested composite structures, as validated by the experiments. Although some small differences exist between the results of FE calculations and experiments, the FE analysis can provide a whole picture and elucidate the changes in the Poisson's ratio and mechanical properties of composite structures with variations in mechanical properties of the constituent materials.

(4) The Poisson's ratio of the proposed composite material depends on its internal structure and mechanical 
properties of constituents. For a given geometry of the composite, the contrast in stiffness of constituents dominates the deformation behavior and mechanical performance of the composite material. Its Poisson's ratio can be reduced with the increase in the stiffness of inclusions, and can be changed from positive to negative if the contrast in stiffness of constituent materials is sufficiently high. In this study, when the material for inclusions was 100 times stiffer than matrix, a strong NPR effect was achieved. Moreover, the effect was independent of the linearity of the materials. Therefore, this kind of composite could be fabricated using both linear and nonlinear materials.

Acknowledgements The author would like to thank the funding support from the Research Grants Council of Hong Kong Special Administrative Region Government in the form of a GRF project (grant no. 515812).

\section{References}

1. Liu Y, Hu H (2010) Sci Res Essays 5:1052

2. Prawoto Y (2012) Comp Mater Sci 58:140

3. Lakes RS, Witt R (2002) Int J Mech Eng Educ 30:50

4. Lakes R (1987) Science 238:551

5. Evans KE, Alderson KL (2000) Eng Sci Educ J 9:148

6. Evans KE, Alderson A (2000) Adv Mater 12:617

7. Grima JN, Daphne A (2010) Phys Status Solidi (b) 248:111
8. Soman PP, Fozdar DY, Lee JW et al (2012) Soft Matter 8:4946

9. Olympio KR, Gandhi F (2009) J Intell Mater Syst Struct 21:1737

10. Wang ZY, Hu H (2013) Text Res J. doi:10.1177/ 0040517512449051

11. Liu Y, Hu H, Lam JKC et al (2010) Text Res J 80:856

12. Ge Z, Hu H (2013) Text Res J 83:543

13. Hu H, Wang Z, Liu S (2011) Text Res J 81:1493

14. Ma Z, Bian H, Sun C et al (2010) Proceedings of the 2009 ground vehicle systems engineering and technology symposium (GVSETS) 1

15. Scarpa F, Yates JR, Ciffo LG et al (2002) J Mech Eng Sci 216:1153

16. Friis EA, Lakes RS, Park JB (1988) J Mater Sci 23:4406. doi:10. 1007/BF00551939

17. Alderson A, Alderson KL (2007) J Aerosp Eng 221:565

18. Yang W, Li Z, Shi W et al (2004) J Mater Sci 39:3269. doi:10. 1023/B:JMSC.0000026928.93231.e0

19. Kaminakis NT, Stavroulakis GE (2012) Compos B 43:2655

20. Scarpa F, Blain S, Lew T et al (2007) Compos A 38:280

21. Yang DU, Lee S, Huang FY (2003) Finite Elem Anal Des 39:187

22. Mitschke H, Schwerdtfeger J, Schury F et al (2011) Adv Mater 23:2669

23. Fozdar DY, Soman PP, Lee JW et al (2011) Adv Funct Mater 21:2712

24. Xu B, Arias F, Brittain ST et al (1999) Adv Mater 11:1186

25. Lakes R (1987) Science $235: 1038$

26. Alderson A, Evans KE (2002) Phys Rev Lett 89:1

27. Miller W, Ren Z, Evans KE (2012) Compos Sci Technol 72:761

28. Miller W, Hook PB, Smith CW et al (2009) Compos Sci Technol 69:651

29. Bertoldi K, Reis PM, Willshaw S et al (2010) Adv Mater 22:361

30. Hou X, Hu H, Silberschmidt V (2012) Compos Sci Technol $72: 1848$ 